\title{
CONTRIBUCIÓN DE LA GANADERÍA EXTENSIVA AL MANTENIMIENTO DE LAS FUNCIONES DE LOS ECOSISTEMAS FORESTALES
}

\author{
Pilar Fernández Rebollo ${ }^{1}$, M. Dolores Carbonero Muñoz ${ }^{2}$ y Alma García Moreno ${ }^{3}$
}

${ }^{1}$ Escuela Técnica Superior de Ingeniería Agronómica y de Montes. Departamento de Ingeniería Forestal. Universidad de Córdoba. Edificio Leonardo da Vinci. Campus Universitario de Rabanales. Crta. MadridCádiz Km. 396. 14071-CÓRDOBA (España). Correo electrónico: ir1ferep@uco.es

${ }^{2}$ IFAPA. Área de Producción Agraria. Centro Hinojosa del Duque. Junta de Andalucía. Crta El Viso km 15. 14270HINOJOSA DEL DUQUE (Córdoba, España). Correo electrónico: mariad.carbonero@juntadeandalucia.es

${ }^{3}$ IFAPA. Área de Producción Ecológica y Recursos Naturales. Centro Alameda del Obispo. Junta de Andalucía. Avda. Menéndez Pidal s/n. 14004-CÓRDOBA (España). Correo electrónico: alma.garcia@juntadeandalucia.es

\section{Resumen}

Los ecosistemas forestales contribuyen al abastecimiento de alimentos y productos a la sociedad y proporcionan servicios culturales. Además, en los últimos años, la población ha tomando conciencia del papel que desempeñan en la regulación de muchos fenómenos y comienza a demandar de ellos nuevos servicios como el mantenimiento de los recursos genéticos, la fertilidad del suelo o la regulación del clima. No cabe duda que la ganadería extensiva, caracterizada entre otros aspectos por el aprovechamiento a pastoreo de hierbas y pastos leñosos que, sin su concurso, quedarían infrautilizados, amplía la capacidad de los ecosistemas forestales en la provisión de alimentos. Y tampoco cabe duda que muchos paisajes, rasgos y tradiciones culturales ligados a los ecosistemas forestales tienen sus raíces en el propio ganado y su gestión. Pero además, participa de los nuevos servicios de regulación a través del mantenimiento de distintas funciones ecosistémicas. Este trabajo repasa algunos aspectos de la contribución de la ganadería extensiva al mantenimiento de la integridad de los ecosistemas y de sus funciones, focalizando en algunas funciones de regulación: la regulación del ciclo del agua y del clima, el mantenimiento de suelos fértiles y de los de recursos genéticos y la defensa frente a los incendios.

Palabras clave: Carbono en suelo, Ciclo hidrológico, Ciclo de nutrientes, Diversidad funcional, Pastoreo, Prevención de incendios

\section{INTRODUCCIÓN}

Los ecosistemas forestales contribuyen al abastecimiento de alimentos y otros productos a la sociedad y proporcionan servicios culturales. Además, en los últimos años, la población ha tomando conciencia de la importancia de algunas funciones de regulación y comienza a demandar de los ecosistemas nuevos servicios tales como el mantenimiento de los recursos genéticos, la regulación del clima y del ciclo del agua o la formación y mantenimiento de suelos fértiles.

No cabe duda que la ganadería extensiva vinculada a los ecosistemas forestales amplía la capacidad que tienen éstos en la provisión de alimentos y otros productos de origen animal y 
que muchos rasgos y tradiciones culturales ligados a los ecosistemas forestales tienen sus raíces en el propio ganado y su gestión. No en vano, el pastoreo del ganado ha sido una práctica secular en los ecosistemas de la Península Ibérica que ha configurado nuestros paisajes y ha determinado el proceso evolutivo global de nuestra flora y fauna. Pero además, participa de los otros servicios que nos proporcionan los ecosistemas, como son los servicios de regulación de los ciclos biogeoquímicos a través del mantenimiento de distintas funciones ecosistémicas.

Tres aspectos básicos caracterizan a la ganadería extensiva. El primero de ellos tiene que ver con su vinculación a los ecosistemas; son sistemas ligados al territorio en los que el ganado hace uso de los pastos. Sin el concurso del ganado la producción vegetal de muchos ecosistemas tendría mucha menos utilidad para el hombre. El segundo aspecto guarda relación con la fuerte dependencia de los recursos vegetales para cubrir las necesidades alimenticias del ganado, aunque ésta nunca llega a ser completa. En la mayoría de los sistemas extensivos se recurre a alimentos complementarios externos para conseguir determinados objetivos de producción y bienestar animal, bien cuando la producción es escasa o cuando su calidad insuficiente. La dependencia de la producción vegetal de los ecosistemas es, por tanto, variable y guarda relación con las características del propio ecosistema, las condiciones meteorológicas y los objetivos de producción ganadera. Así por ejemplo, en un trabajo realizado en el Parque Natural de Cabo de Gata sobre 10 ganaderías de ovino vinculadas a este espacio natural, se obtuvo que, de media, los ganaderos cubrían con los pastos el $67 \%$ de las necesidades energéticas de sus rebaños, completando con alimentos comprados el 33\% restante (Terradillos, 1997). El nivel de complementación entre ganaderías fluctuó desde el $20 \%$ hasta el $70 \%$ en un mismo año, poniendo de manifiesto la existencia de un amplio gradiente de vinculación con el territorio y sus pastos. En las dehesas andaluzas el porcentaje de las necesidades energéticas cubiertas por los pastos se sitúa en torno al $60 \%$ para el ganado vacuno de cría y al $80 \%$ para el ovino de carne (FERNÁNDEZReBollo, datos no publicados). Por último, el tercer aspecto se refiere a la adaptación del ganado a los ecosistemas forestales. España cuenta con un amplio catálogo de razas autóctonas perfectamente adaptadas a los diferentes ecosistemas de la Península, al que se han incorporado con éxito otras razas foráneas. Las razas bovinas Charolesa, Limusina, Parda; las ovinas Berrichon Du Cher, Ile de France, Fleischaf, Landschaff o Merino Precoz, o el ganado porcino de raza Duroc, son algunos ejemplos. Por tanto, los sistemas ganaderos extensivos vinculados a los ecosistemas forestales no están exclusivamente asociados a las razas autóctonas.

En los últimos años, la baja rentabilidad de la ganadería extensiva está motivando el abandono del pastoreo en muchos ecosistemas forestales. Al mismo tiempo, el proceso de intensificación de algunos sectores ganaderos, como por ejemplo el ovino lechero, ha llevado a una mayor desvinculación del territorio donde el aporte de alimento en pesebre predomina sobre el pastoreo. El abandono del pastoreo implica cambios en la estructura y funcionamiento de los ecosistemas y, por tanto, en los servicios que nos proporcionan, no solo aquellos culturales o de abastecimiento de productos, sino también en los servicios de regulación.

Este trabajo pretende repasar algunos aspectos de la contribución de la ganadería extensiva al mantenimiento de la integridad de los ecosistemas y a su capacidad de generar funciones, focalizando principalmente en algunas funciones de regulación: la regulación del ciclo del agua, el mantenimiento de suelos fértiles, el mantenimiento de recursos genéticos, la regulación climática y la defensa frente a grandes perturbaciones como son los incendios.

\section{EL CICLO DEL AGUA EN LOS ECOSISTEMAS TERRESTRES Y LOS EFECTOS DE LA GANADERÍA EXTENSIVA}

El pastoreo del ganado puede afectar a distintas fases del ciclo hidrológico condicionando a pequeña escala el reparto del agua a lo largo del territorio y, por extensión, la erosión que puede sufrir el suelo debida a su flujo. Aunque la partición del flujo del agua en los ecosistemas viene determinada en primera instancia por el 
relieve, el tipo de vegetación y las características edáficas, el ganado a pastoreo, por su capacidad de alterar la estructura, la composición y la tasa de crecimiento de la vegetación y las características edáficas, puede condicionar la dinámica del agua en el ecosistema y el papel que pueda tener ésta en la erosión del suelo.

La interceptación de la precipitación en una determinada zona, es decir, la cantidad de agua de lluvia que puede llegar al suelo, depende entre otros factores del tipo de vegetación presente. El pastoreo del ganado al modificar la estructura y la composición de la vegetación puede, a largo plazo, afectar a la cantidad total de agua interceptada y a la energía cinética con la que el agua llega al suelo. No obstante en muchas situaciones, la actuación del hombre sobre la vegetación (para fomentar plantas más palatables o estructuras vegetales con mayor eficiencia de cosecha) tiene un impacto mayor sobre la interceptación que la del ganado.

El ganado puede tener un efecto mayor sobre la retención del agua en la superficie del suelo al modificar la rugosidad del terreno. No obstante, el efecto del ganado en la rugosidad va a depender de la densidad de la vegetación herbácea y de sus raíces, de la textura y humedad del suelo y de la carga ganadera (DREWY, 2006). Así, una carga ganadera baja en suelos húmedos puede dar lugar a un incremento del microrelieve aumentando la retención del agua, mientras que una carga ganadera alta puede originar una reducción de la rugosidad por una disminución del mantillo y restos vegetales y de los agregados del suelo, dando lugar a un mayor flujo superficial tras un evento de lluvia. No obstante, otras características químicas y biológicas del suelo parecen afectar también al flujo superficial del agua compensando los efectos de la rugosidad del terreno. Así por ejemplo, Schnabel et al. (2013) han encontrado una relación negativa entre la superficie desprovista de vegetación y la cualidad del suelo de repeler agua. En un muestreo amplio realizado en dehesas extremeñas, estos autores encuentran que la cualidad hidrofóbica de un suelo esta relacionada con el tipo de cobertura en la superficie, siendo suelos hidrofóbicos aquellos cubiertos por hojarasca de encina e hidrofílicos los que presentan mayor porcentaje de suelo desnudo. Dado que en las dehesas la presencia de suelo desnudo está relacionada con una mayor densidad de ganado, ésta reduce no solo la rugosidad del suelo sino también la cualidad de repeler el agua cuando se parte de una situación seca.

La tasa de infiltración del agua puede verse afectada por el pastoreo del ganado, habiéndose destacado el papel de la compactación producida por el pisoteo continuado.

El pisoteo del ganado compacta el suelo reduciendo el volumen de poros que queda entre los agregados, dando lugar a un aumento de la densidad aparente (KULLI et al., 2003, DreWY et al., 2008). De forma natural y fruto de los distintos procesos de formación, un suelo puede ver reducido su volumen de poros, diciéndose en estos casos que se ha producido una consolidación. Ésta se diferencia de la compactación en el origen de las cargas: naturales en el primer caso, de origen antrópico en el segundo.

La compactibilidad de un suelo depende de la textura, del contenido en materia orgánica y de la humedad (BILOTTA et al., 2007; GREENWOOD \& MCKENZIE, 2001). La textura del suelo determina el número y tamaño de los poros y por tanto la disminución de la porosidad causada por fuerzas externas. La textura que en teoría es más susceptible a la compactación se encuentra en la clase limo arenoso, ya que las partículas de distintos tamaños se pueden acomodar mejor en un espacio más reducido. Por otro lado, la materia orgánica del suelo aumenta la estabilidad de los agregados y por tanto la capacidad del suelo de soportar una fuerza externa (FRANKZLUEBBERS \& Studemann, 2008; SoAne, 1990). Además, la acumulación de materia orgánica en la superficie del suelo puede atenuar las fuerzas externas actuando a modo de colchón (DONKOR et al., 2002; SOANE, 1990).

La presión ejercida por la pezuña del ganado sobre la superficie del suelo puede ser de la misma magnitud que la producida por la maquinaria. Por ejemplo, la presión bajo la pezuña de una oveja ronda los 50-80 kPa (BILlOTA et al., 2007), valor comparable a los $60-80 \mathrm{kPa}$ bajo la rueda de un tractor de gomas (BLUNDEN et al., 1994). Sin embargo, la presión ejercida por el animal puede ser superior cuando está en movimiento y transitando por zonas con cierta pendiente, debido a la energía cinética y a la transferencia 
del peso del animal al extremo de las pezuñas cuando se mueve hacia arriba o hacia abajo.

En general, y a diferencia de lo que ocurre con los vehículos y la maquinaria, la compactación producida por el pisoteo del ganado solo afecta a la superficie del suelo (BELL et al., 2011; BilotTA et al., 2007; FERnÁNDEZ ReBOllo et al., 2004). Bajo una misma presión superficial, la distribución de la compactación con la profundidad depende del área de contacto y a medida que ésta se reduce, la compactación queda confinada en la superficie. Por tanto, la compactación producida por el tránsito de pequeños rumiantes (ovino y caprino) es más superficial que la debida al pisoteo del vacuno. En todo caso, es poco frecuente que la compactación debida al ganado se extienda más allá de los 10 primeros $\mathrm{cm}$ del suelo (DANIEL et al., 2002; DONKOR et al., 2002; FERNÁNDEZ RebOllo et al., 2004). Además se produce con el pisoteo inicial del ganado, alcanzándose tras poco tiempo de pastoreo, valores máximos de acuerdo a la presión ejercida y a la compactibilidad del suelo.

Otra característica a resaltar de la compactación producida por el ganado, radica en que la reducción del volumen de poros afecta principalmente a los macroporos. En algunos casos se ha sugerido un efecto compensatorio en la distribución del tamaño de los poros como respuesta al pisoteo del ganado, en donde una disminución del volumen de macroporos lleva parejo un aumento del volumen de microporos y por tanto la porosidad total del suelo no variar (HoUlBROOKE et $a l .$, 2011). Esto puede explicar que en los muestreos que venimos realizando en dehesas con un gradiente de carga ganadera no encontremos diferencias en la densidad aparente del suelo (datos no publicados). En este sentido, la contribución de la materia orgánica del suelo puede ser determinante en la construcción de los microporos (STOCK \& DownES, 2008).

En definitiva, el pisoteo del ganado disminuye el volumen total de poros en la superficie del suelo y/o la distribución de sus tamaños, reduciendo la tasa de infiltración lo que puede derivar en mayor escorrentía a escala de cuenca (DrEwry et al., 2008). Un aumento de la carga ganadera y del pisoteo se ha asociado con una disminución mayor de la tasa de infiltración (BELl et al., 2011; GREENWOOD \& MCKENZIE,
2001), pero también con una mayor capacidad para retener agua en el horizonte superficial especialmente en suelos de textura arenosa-, lo que puede incrementar en algunos casos la producción de las especies vegetales con sistemas radicales someros y/o alargar su periodo de crecimiento (HoulbrooKe et al., 2011; Drewry et al., 2008; DoNKOR et al., 2002). No obstante, la información disponible parece indicar que, en general, la producción vegetal disminuye con el aumento de la compactación y que es la dureza del suelo (BELL et al., 2011; STAVI et al., 2011) más que la modificación de la porosidad, el factor que limita el crecimiento, al suponer un freno a la elongación de las raíces (GREENWOOD \& MCKenZie, 2001). Por ejemplo, Bengough et al. (2011), demuestran que durezas del suelo entre 0,8-2 MPa limitan el crecimiento de las raíces y MATERECHERA et $a l$. (1991), indican que éste se impide totalmente cuando la dureza del suelo es superior al rango 3,6-5,1 $\mathrm{MPa}$.

Un suelo compactado por el ganado puede volver a recuperarse, jugando un papel importante en este proceso la actividad biológica de los organismos del suelo (DREWRY, 2006; KULLI et al., 2003), y la acción de las raíces (FRANZLUEBBERS Y STUEDEMANN, 2008; GREENWOOD \& MCKENZIE, 2001). Así por ejemplo, se ha encontrado una relación positiva entre la biomasa de raíces y la porosidad (Houlbrooke et al., 2011; STAVI et al., 2011; BeLL et al., 2011), y un aumento de la estabilidad estructural del suelo por el ensamblaje entre los agregados del suelo y las raíces finas (StAVI et al., 2011; BiLOTTA et al., 2007; GreENWOOD \& MCKenZIE, 2001). El tiempo requerido para recuperar un suelo compactado depende de muchos factores entre los que se han citado el tipo de suelo y de vegetación, la severidad de la compactación y las condiciones climáticas. Por ejemplo, Drewry et al. (2004) indican que en Nueva Zelanda un suelo compactado por ganado vacuno lechero durante el periodo húmedo, se recupera evitando el pastoreo en verano y otoño. En climas más secos puede ser necesario mayor tiempo (GREENWOOD \& MCKENZIE, 2001).

El ganado a pastoreo puede en algunos casos aumentar la tasa de transpiración global de la vegetación como consecuencia de un mayor crecimiento debido a la aceleración del ciclo de los nutrientes (GARCíA-MORENO et al., 2014), aspec- 
to que se discutirá en el siguiente apartado. Pero, al defoliar de forma selectiva, altera también la tasa de transpiración relativa de las distintas especies o grupos de especies. Es decir, aquellas especies vegetales más consumidas verán reducida su superficie foliar y por tanto su tasa de transpiración a coroto plazo en beneficio de las menos consumidas que podrán disponer de mayor cantidad de agua. De esta forma, el empleo del ganado para reducir la competencia por agua entre la vegetación herbácea y el árbol ha sido una práctica muy extendida en los frutales de secano, revitalizada actualmente en aquellas zonas donde la orografía del terreno dificulta la mecanización y desaconseja otras alternativas (laboreos o siegas) (CARBONERo et $a l ., 2013$ ) y extrapolada a plantaciones forestales. Por último, aunque algunos trabajos indican que el agua se evapora más rápidamente desde suelos compactados que desde aquellos con una buena estructura, la relación entre pastoreo y evaporación del agua desde el suelo no está clara.

$\mathrm{La}$ información disponible parece indicar que en los terrenos pastoreados aumenta la cosecha de agua por escorrentía y se reduce el drenaje en profundidad. No obstante, a nivel de cuenca estos flujos pueden verse compensados si existen gradientes de pastoreo. En todo caso el papel del ganado en la modificación del ciclo del agua deriva de una compleja interacción de múltiples factores relacionados con su impacto sobre la vegetación, y sobre las propiedades físicas y químicas del suelo, que aun no están completamente esclarecidas.

\section{EL PAPEL DE LA GANADERÍA EXTENSIVA EN EL FOMENTO Y CONSERVACIÓN DE LA FERTILIDAD DEL SUELO}

En general, se acepta que el pastoreo modifica el ciclo de los nutrientes acelerando la tasa de conversión desde formas orgánicas hacia formas inorgánicas. Este proceso de mineralización puede ser crítico para la producción vegetal dado que en muchos ecosistemas un gran porcentaje de los nutrientes esenciales para el crecimiento de las plantas se encuentran en formas orgánicas.
El ganado aumenta la mineralización por dos vías. En primer lugar, reduciendo el tamaño de las partículas del material vegetal que consume mediante la masticación inicial y, en el caso de los ruminates, la rumia posterior. El pisoteo del ganado también realiza una importante labor de disminución del tamaño de las partículas vegetales y mezcla posterior con el suelo, aunque en este caso el fraccionamiento sea menos intenso que el realizado a través de la masticación.

En segundo lugar, y en el caso también de los ruminates, creando en el rumen un ambiente favorable para el desarrollo de una densa flora microbiana que degrada el material vegetal consumido para obtener la energía y la proteína necesaria para su propio crecimiento. Dado que el ganado retiene sólo una pequeña parte de los nutrientes consumidos, éstos vuelven al ecosistema en forma de heces y orina tras haber sufrido una intensa degradación a lo largo del tracto digestivo del ganado.

Los nutrientes excretados en la orina (principalmente nitrógeno, potasio, magnesio y azufre) se encuentran en forma inorgánica y por tanto disponibles para las plantas. Por el contrario, una proporción importante de los nutrientes contenidos en las heces sólidas están en forma orgánica y deben ser transformados por los microorganismos del suelo previamente a su utilización por las plantas. Por tanto, en los ecosistemas con presencia de ganado a pastoreo, una proporción importante de los nutrientes ingeridos son devueltos rápidamente al ecosistema en formas inorgánicas. La mayor concentración de nutrientes en la vegetación de zonas pastoreadas versus zonas no pastoreadas, apoya esta hipótesis de mayores tasas de recirculación de nutrientes. Así, García-Moreno et al. (2014), en un trabajo desarrollado en dehesas de la provincia de Córdoba encuentran una relación positiva entre la concentración de nitrógeno y magnesio foliar en la encina y la intensidad de pastoreo.

La concentración de nutrientes de la excreta del ganado no es constante sino que varía a lo largo del año y entre años en función de la disponibilidad de pastos, de su concentración en nutrientes y su digestibilidad. Por ejemplo, en pastos herbáceos anuales mediterráneos las excretas del ganado de finales de otoño y de invierno suelen presentar una mayor concentra- 
ción de nutrientes que las de finales de primavera o verano, debido a la mayor riqueza de nutrientes y digestibilidad de la hierba en estos momentos. Y es precisamente al final del invierno cuando estos pastos herbáceos demandan una mayor cantidad de nutrientes para su crecimiento. Las excretas del ganado pueden, por otro lado, alterar las tasas de mineralización que sufren los nutrientes orgánicos en el suelo. Por ejemplo, Augustine et al. (2006), estudiando la dinámica del nitrógeno en pastos africanos, encuentran que el pastoreo de los grandes herbívoros aumenta la cantidad de nitrógeno del suelo en forma inorgánica durante la estación seca y disminuye la tasa de mineralización del nitrógeno orgánico del suelo al comienzo de la estación de crecimiento de la vegetación. Estos resultados sugieren que la disponibilidad de nitrógeno para la vegetación durante la estación de crecimiento está controlada por el balance entre el efecto positivo de los herbívoros sobre el contenido de nitrógeno inorgánico en el periodo seco y el efecto negativo posterior en la tasa de mineralización. Por tanto, el ganado y los grandes herbívoros más que el pastoreo en sí mismo, parecen determinar la dinámica del nitrógeno a corto plazo en muchos ecosistemas, poniendo de manifiesto que juegan un papel dual al actuar como consumidores y descomponedores.

Asimismo las excretas del ganado pueden aumentar temporalmente el $\mathrm{pH}$ del suelo, mejorando la solubilidad de algunos nutrientes especialmente en suelos ácidos. Así, en suelos de dehesas LeCHUGA (2005) encuentra una disminución significativa de la acidez del suelo tras dos noches de concentración de ganado ovino en una parcela pequeña, pasando el $\mathrm{pH}$ del suelo (medido en los 10 primeros $\mathrm{cm}$ ) de 6,0 a 7,2.

Conviene resaltar dos aspectos relacionados con el pastoreo del ganado y su repercusión en la fertilidad de los suelos. El primero de ellos tiene que ver con el reparto espacial de los nutrientes excretados por el ganado. Se asume que el ganado puede cosechar pasto de una superficie amplia pero las excretas quedan distribuidas en una superficie menor lo que genera a pequeña escala parches con distinta fertilidad y a nivel de paisaje la movilización de nutrientes entre zonas (los altos frente a los bajos) o entre comunidades vegetales. La distribución de las excretas depende de las comunida- des vegetales presentes (principalmente por la cantidad y calidad de alimento que pueden proporcionar), de los hábitos de pastoreo de las distintas especies y razas ganaderas (ARCHER, 1995), de su experiencia en pastoreo y del número y localización de los elementos que atraen al ganado (puntos de agua, refugios, comederos). La distribución de las excretas tiende a ser más homogénea cuando el pastoreo se realiza con pequeños rumiantes, y en todo caso, siempre es más uniforme el reparto de la orina. La agrupación del ganado durante la noche en corrales al aire libre concentra las excretas y promueve, tras su abandono, el desarrollo de comunidades vegetales específicas. Baste como ejemplo el redileo con ovino en el mediterráneo, que genera los pastos conocidos como majadales, comunidad vegetal dominada por gramíneas de talla baja (principalmente Poa bulbosa) con algunas leguminosas (Trifolium subterraneum o Medicago spp.) y otras plantas herbáceas de hoja ancha. Pero también otras prácticas como las "bomas", o corrales en los que los pastores nómadas africanos concentran el ganado vacuno y que, tras su abandono, son colonizadas por una comunidad vegetal dominada por gramíneas de talla baja (Cynodon plectostachyus y Sporobolus pellucidus) con algunas ciperáceas y plantas de hoja ancha (AUGUSTINE, 2003a). Más allá de la necesidad de controlar al ganado y de proveer refugio frente a predadores, en muchas dehesas y rastrojeras se concentra deliberadamente al ganado ovino como vía para mejorar la fertilidad del suelo.

El segundo aspecto guarda relación con la permanencia en el tiempo de la fertilidad del suelo inducida por el pastoreo y de las comunidades vegetales que promueve. Algunos trabajos revisados inducen a pensar que estas modificaciones pueden permanecer durante mucho tiempo en el paisaje. Así por ejemplo, en el trabajo de LECHUGA (2005) antes comentado, la diferencia en el $\mathrm{pH}$ del suelo entre la zona pastoreada y la zona donde el ovino estuvo concentrado dos noches consecutivas se mantiene significativa un año después de la pernocta; o por ejemplo "bomas" abandonadas hace más de 40 años que aún muestran suelos enriquecidos en carbono total, nitrógeno y fósforo pudiendo perdurar estas condiciones posiblemente durante siglos si regularmente siguen siendo pastoreadas de forma extensiva (AUGUSTINE, 2003b). 
Por tanto, el ganado aumenta el ritmo de recirculación de los nutrientes pero al mismo tiempo la probabilidad de ser perdidos del ecosistema. La pérdida de nutrientes de los ecosistemas ocurre principalmente por volatilización, percolación, erosión del suelo o bien por la extracción de los productos ganaderos. En general, el pastoreo del ganado no aumenta considerablemente las pérdidas de nutrientes de los ecosistemas creando un balance negativo, salvo que exista erosión del suelo. La fijación de nitrógeno atmosférico junto al aumento de la tasa de recirculación de nutrientes parecen suficientes para compensar las pérdidas debidas al pastoreo en muchos ecosistemas utilizados de forma extensiva. Además, las reservas del suelo en materia orgánica pueden amortiguar las pérdidas de nutrientes a corto plazo debidas a periodos secos o a presiones puntuales de pastoreo elevadas. Por otro lado, las pérdidas de nutrientes debido a la extracción de los productos ganaderos no son importantes y están minimizadas por la baja productividad de muchos de nuestros ecosistemas y por la propia fisiología del aparato digestivo de los rumiantes. Además, la complementación al pastoreo, práctica habitual en la gestión del ganado, constituye una vía adicional de entrada de nutrientes al ecosistema compensando con creces las salidas en forma de productos ganaderos (GARCíA-Moreno et al., 2013).

Los efectos sobre la disponibilidad de nutrientes en el suelo mediados por el ganado, junto a los mecanismos de respuesta de las plantas que operan a escala de individuo o de ecosistema, han sustentado la hipótesis de la "optimización del pastoreo", que sugiere que un pastoreo moderado puede potencialmente aumentar la producción primaria de un ecosistema sobre la que tendría en ausencia del mismo. Aunque existen algunas evidencias que apoyan esta hipótesis, la variabilidad climática condiciona la relación planta-herbívoro, con unos efectos negativos en la producción los años secos independientemente de la disponibilidad de nutrientes en el suelo (AIREs et al., 2008). Además, otros trabajos sugieren que en determinados ecosistemas el pastoreo del ganado y de los grandes herbívoros puede producir una desaceleración en el ciclo de los nutrientes reduciendo la producción primaria del ecosistema. Esto puede suceder en ecosistemas donde el pastoreo selectivo de lugar a un aumento de especies de baja palatabilidad, leñosas o con muchos mecanismos de defensa (ANDERSON \& BRISKE, 1995). El pastoreo selectivo puede limitar también la abundancia de especies fijadoras de nitrógeno, un grupo funcional clave en muchos ecosistemas (RITCHIE et al. 1998; LEE et al., 2003).

\section{LA GANADERÍA EXTENSIVA Y LA DIVERSIDAD VEGETAL DE LOS ECOSISTEMAS}

Los paisajes vegetales que han llegado hasta nuestros días son fruto del pastoreo secular del ganado y de los grandes herbívoros, los cuales han contribuido a su diversificación y mantenimiento. Se asume que los factores abióticos son los principales responsables de la variación de la vegetación a escala de paisaje al que le sigue, según algunos autores, el pastoreo del ganado y la gestión de los pastores que, difícilmente, puede disociarse de los efectos del ganado (Sebastia et al., 2008; 2011).

Muchos de los sistemas valorados positivamente por la elevada diversidad que contienen, son fruto de la gestión del hombre en beneficio del ganado, la agricultura o la provisión de combustible. Quizá uno de los mayores exponentes lo tenemos en la dehesa con una riqueza muy elevada en sus pastos herbáceos (MARAÑón, 1986), derivada de la propia heterogeneidad espacial y temporal de las características edáficas y climáticas, a la que se le superpone las prácticas agrícolas (barbechado del suelo, laboreo para el establecimiento de cultivos agrícolas, estercolados, huertos, setos, cercas de piedra...), selvícolas (reducción de la densidad, podas del arbolado, desbroces, carboneo...) y, sobre todo, el pastoreo del ganado. El pastoreo mixto que se ha desarrollado en la dehesa combinando en la misma explotación distintas especies ganaderas y razas, ha podido tener también un papel relevante en los patrones de los pastos herbáceos. En el sudeste peninsular, ROBLES (2008) indica que los ecosistemas arbustivos abiertos con mayores posibilidades de uso pastoral son los que presentan mayor diversidad florística. Por ejemplo, esta autora destaca a los aulagares y los lentiscares 
abiertos que presentan una diversidad superior a 2 bits sin contabilizar las especies herbáceas. En pastos pirenaicos, de Bello et al. (2006) han encontrado además una variación en la diversidad florística en función de la presión ganadera. La mayor diversidad de los pastos herbáceos se encontraba en las zonas con mayor presión pastoral mientras que los pastos con menor uso ganadero o abandonados presentaban una composición menos variada. Además, la especie ganadera puede condicionar la distribución de las distintas comunidades vegetales, estando relacionado el pastoreo del ganado ovino con una mayor diversificación y heterogeneidad a nivel de paisaje (ALDEZABAL et al., 2002).

Sin embargo, abundan también los trabajos en donde no se constata una variación importante en la diversidad florística como respuesta al pastoreo, entendida ésta cómo número de especies. Pero aunque no haya variación en el número si se produce un cambio importante en la composición en especies. Por ejemplo, PECO et al. (2006), comparando pastos de la Sierra de Guadarrama pastoreados y abandonados hace treinta años, no encuentra diferencia en el número de especies presentes pero si encuentra un cambio drástico en la composición: ambas zonas comparten sólo el $40 \%$ de las especies y el $60 \%$ restante son exclusivas de las zonas pastoreadas o bien de las zonas abandonadas. TARREGA et $a l$. (2009), en pastos de dehesas de $Q$. pyrenaica en la provincia de León, tampoco encuentra diferencias en el número de especies a nivel de parcela o de comunidad tras el abandono del pastoreo, aunque si disminuye el número de especies herbáceas anuales.

Por tanto el pastoreo, más que a la riqueza florística en sí, afecta a la diversidad funcional, entendida ésta como el conjunto total de caracteres funcionales de la vegetación y su abundancia (DíAZ et $a l ., 2007$ ). El funcionamiento de un ecosistema está más relacionado con los caracteres funcionales de las distintas especies que con la riqueza florística y, aunque todas y cada una de las especies juegan un papel en el funcionamiento de un ecosistema, la naturaleza y magnitud de las contribuciones individuales varían considerablemente según el ecosistema o el proceso considerado. Así por ejemplo en el trabajo antes comentado de BeLlo et al. (2006), al mismo tiempo que aumenta la diversidad florística con la presión ganadera se produce una reducción de la diversidad funcional, pues aumentan las especies redundantes con similar papel en el ecosistema. Tratar de entender qué rasgos funcionales de la vegetación favorece el pastoreo del ganado ha sido y es el objetivo de muchos trabajos.

El ganado puede controlar la dinámica de colonización-extinción de las especies favoreciendo a aquellas con determinados rasgos funcionales a través de distintos mecanismos (OLFF \& RitchiE, 1998) que afectan a los individuos directamente e indirectamente (BRISKE, 1996). Los efectos directos están asociados con las alteraciones que la defoliación y el pisoteo producen en la fisiología y morfología de las distintas especies vegetales, mientras que los efectos indirectos se derivan de las modificaciones inducidas por el pastoreo sobre el microclima, las propiedades físicas, químicas y biológicas del suelo que alteran la capacidad competitiva de cada especie. El ganado realiza también una importante labor de distribución de semillas (ectozoocoría y endozoocoría) incorporando otras especies, en muchos casos mejorando incluso el éxito de la germinación tras su paso por el tracto digestivo (Herrera, 2000; Castro y Robles, 2003).

Pues bien, a pesar de la cantidad de trabajos publicados que abordan la respuesta de las especies vegetales al pastoreo y la dinámica de la vegetación, no hay un modelo general válido que permita predecirla. Siguiendo a BRISKE (1996), los modelos aceptados son extrapolaciones de estudios locales y por tanto la validez de los rasgos y atributos vegetales que pueden ser favorecidos por el pastoreo no han sido contrastados a nivel global.

Así por ejemplo, es clásico el modelo "rangesucession" de DYKSTERHUIS (1949) el cual predice como respuesta al pastoreo un aumento de las plantas anuales, una disminución de las perennes, un desplazamiento de las plantas palatables por otras de menor palatabilidad y una sustitución de los pastos dominados por gramíneas altas por pastos dominados por gramíneas de talla baja, matorrales y plantas de hoja ancha de hábitos rastreros. Este modelo constituyó la base para la gestión de los pastos americanos y supuso el desarrollo de protocolos técnicos para evaluar la condición de los pastos y la tendencia de los 
cambios observados tras el pastoreo, algunos de los cuales aún están en vigor.

Posteriormente, MiLCHUNAs et al. (1988) se centran en la idea de que los rasgos de la vegetación como respuesta al pastoreo pueden variar dependiendo del régimen de precipitación y de la historia de pastoreo. Asumen que la aridez y el pastoreo desencadenan en la vegetación respuestas similares, dado que las adaptaciones que mejoran la supervivencia en ambientes áridos promueven al mismo tiempo la tolerancia o la defensa frente al pastoreo. Es decir, el estrés hídrico y el estrés producido por el pastoreo son similares en el sentido de que ambos implican una reducción del tejido vegetal. Por el contrario, en ambientes más húmedos, la competencia entre especies, principalmente por la luz, y la tolerancia al pastoreo desencadenan rasgos vegetales divergentes que permiten modos de competencia dependientes de la intensidad de pastoreo. De esta forma, estos autores indican que como respuesta a la intensidad de pastoreo aumentarían las plantas anuales, de talla baja, con hábito de crecimiento postrado, estoloníferas y graminoides. Pero estos cambios serían más marcados en ecosistemas con una larga historia de pastoreo y climas húmedos. En cambio, en ecosistemas donde el pastoreo del ganado es reciente un aumento de la presión de pastoreo puede dar lugar a la invasión de especies exóticas, especialmente en aquellos ambientes más húmedos y productivos.

Para GRIME (2001), el pastoreo favorecería rasgos propios de las especies ruderales (anuales, de pequeña talla y de rápido crecimiento). No obstante, en ambientes áridos los rasgos propios de las especies tolerantes al estrés hídrico también podrían verse favorecidos con el aumento de la presión de pastoreo (talla pequeña, hábito de crecimiento postrado y perenne). La palatabilidad se vería favorecida en ambientes productivos donde los mecanismos de tolerancia al pastoreo promoverían rápidos rebrotes al destinar todos los recursos al crecimiento y pocos a mecanismos de defensa y disminuiría en ambientes más secos, y por tanto menos productivos, en los que las plantas derivarían una parte de sus recursos a la formación de estructuras de defensas en detrimento del crecimiento.

Recientemente DíAz et $a l$. (2007), analizando los rasgos y atributos vegetales inducidos por el pastoreo a partir de 197 trabajos realizados en todo el mundo concluyen que, en general, el pastoreo beneficia a las plantas de pequeña talla, hábito de crecimiento postrado y arquitectura en roseta y estolonífera, y estas respuestas parecen más marcadas en las zonas más productivas (con mayor precipitación) y pastoreo secular. Además, favorece a las plantas anuales en detrimento de las perennes, aunque este patrón no se muestra tan consistente en las zonas áridas con una larga tradición de pastoreo. El pastoreo parece no fomentar a las plantas herbáceas de hoja ancha frente a las de hoja estrecha o viceversa, ya que la respuesta neutral es lo más frecuente y extendido, indicando estos autores que cómo grupos funcionales son muy amplios. Así por ejemplo, las gramíneas amacolladas se ven perjudicadas por el pastoreo mientras que aumenta la abundancia de gramíneas estoloníferas. Además, estos autores tampoco encuentran una respuesta clara de las leguminosas ni de las leñosas como grupos funcionales frente al pastoreo. .A pesar de la abundante literatura que preconiza una matorralización con el aumento del pastoreo en las zonas áridas. Por último, el pastoreo tiende a incrementar las plantas poco palatables principalmente en ambientes áridos con pastoreo secular. Por tanto, además del estado del ecosistema de partida, el contexto histórico y climático ayuda a entender que rasgos y características de las plantas se ven fomentados por el pastoreo en los modelos generales.

El pastoreo extensivo del ganado nunca es uniforme. La propia topografía, la distribución de los tipos de vegetación, la localización de los puntos de agua, los pastores en el caso de conducir el ganado, las condiciones meteorológicas etc, originan gradientes de pastoreo que permiten la coexistencia a escala de paisaje o incluso de parcela (FANLO et $a l .$, 2013) de especies pertenecientes a grupos funcionales no fomentados por el pastoreo del ganado. Los ganaderos y pastores fomentan también estos gradientes al pastorear con distinta presión, en distinto momento o con distintas especies ganaderas. Y esto es una fuente de resiliencia ecológica. La desaparición de la ganadería de algunos ecosistemas forestales podría llegar a amenazar la existencia de especies con rasgos funcionales favorecidos por el pastoreo del ganado. 


\section{LA GANADERÍA EXTENSIVA Y EL SECUESTRO DE CARBONO EN SUELO}

La ganadería extensiva, con un pastoreo gestionado adecuadamente, puede tener un efecto positivo sobre la materia orgánica del suelo (GARCÍA-Moreno et al., 2013; Augustine, 2003b). Ésta, además de tener una función importante en los ecosistemas como reservorio de fertilidad, puede contribuir a la mitigación del cambio climático. No en vano se considera que el carbono orgánico del suelo representa la mayor reserva en interacción con la atmósfera, por encima de la vegetación, aunque con tiempos de residencia variables.

Los estudios que evalúan el balance de carbono en los ecosistemas han puesto de manifiesto que, en general, los pastos aprovechados por grandes herbívoros constituyen un importante sumidero de $\mathrm{CO}_{2}$ atmosférico (Soussana et al., 2007; CERnUSCA et al., 2008), aunque en años secos pueden comportarse como fuente de $\mathrm{CO}_{2}$ (AIRES et al., 2008).

El contenido de carbono orgánico en suelos de distintos pastos de la Península Ibérica se ha estimado en torno a $73 \mathrm{Mg} \cdot \mathrm{C} \cdot \mathrm{ha}^{-1}$, aunque con variaciones en función del tipo de suelo (RodrígueZ-Murillo, 2001). Por ejemplo, este estudio aporta contenidos de $71 \mathrm{Mg} \cdot \mathrm{C} \cdot \mathrm{ha}^{-1}$ en cambisoles y $99 \mathrm{Mg} \cdot \mathrm{C} \cdot \mathrm{ha}^{-1}$ en leptosoles. En dehesas mediterráneas hemos encontrado un contenido similar en cambisoles $\left(76 \mathrm{Mg} \cdot \mathrm{C} \cdot \mathrm{ha}^{-1}\right.$ ) aunque algo menor en leptosoles (58 Mg.C $\cdot \mathrm{ha}^{-1}$ ) debido principalmente a diferencias en la densidad aparente y en el espesor del suelo (PARRASALCÁNTARA et $a l$., en preparación). La cantidad de carbono orgánico acumulado en los pastos es pues muy significativa y comparable en algunas ocasiones a las encontradas en bosques y formaciones forestales. Por ejemplo SedDAIU et al. (2013), comparando el contenido de carbono orgánico en suelos similares pero con distinto uso, encuentran escasas diferencias en la calidad y cantidad de la materia orgánica entre pastos y bosques de Quercus (encinas y alcornoques). Además, estos autores constatan que en los bosques la acumulación de carbono orgánico se produce en un delgado horizonte superficial (4 $\mathrm{cm}$ ) disminuyendo bruscamente con la profundidad en comparación con los pastos, donde ésta ocurre en un horizonte de mayor espesor. Esto podría dotar a los ecosistemas forestales pastoreados de una mayor resiliencia en materia de secuestro de carbono frente a perturbaciones del suelo (FrANZLUEBBERS, 2002).

La acumulación de carbono orgánico en el suelo guarda relación con la producción de la vegetación, principalmente con el desarrollo que pueden llegar a tener las raíces. Los restos de raíces tienden a presentar mayor dificultad para ser descompuestos por los organismos del suelo que las hojas y brotes de las plantas, debido principalmente a su mayor contenido en compuestos recalcitrantes a la degradación como la lignina y los polifenoles (FrANZLUEBBERS et al., 1996). Además, la liberación por parte de las raíces de compuestos orgánicos contribuye a estabilizar los agregados del suelo, protegiendo así a la materia orgánica de la descomposición.

Como hemos visto en el apartado previo, el ganado extensivo puede contribuir a mejorar la productividad de la vegetación a través de sus efectos sobre el reciclaje y mineralización de los nutrientes, especialmente del nitrógeno, elemento que puede limitar el crecimiento de la vegetación en mayor medida. Por ejemplo, una vaca de $500 \mathrm{~kg}$ de peso vivo seca puede ingerir al día alrededor de $280 \mathrm{~g}$ de nitrógeno en un pasto con un $18 \%$ de proteína bruta, una digestibilidad del $65 \%$ y una disponibilidad de hierba en torno a los $1.500 \mathrm{Kg}$ de MS $\cdot \mathrm{ha}^{-1}$, de los cuales devuelve en la orina en forma de urea $220 \mathrm{~g}$ de nitrógeno cada día. Podemos equiparar este efecto del ganado a una fertilización nitrogenada fraccionada en el tiempo y con riqueza variable. Por esta vía se puede incrementar el crecimiento de la vegetación en años de meteorología favorable (AIRES et al., 2008), evitándose al mismo tiempo los elevados costes en términos de carbono que implica la producción y distribución de los fertilizantes nitrogenados. Según Soussana et al., (2010) se pueden obtener incrementos de 0,2 a $0,5 \mathrm{t} \mathrm{C} \cdot$ ha ${ }^{1} \cdot$ año $^{-1}$ mediante una serie de medidas de gestión entre las que se incluyen una intensificación moderada del pastoreo en aquellos ecosistemas de zonas templadas pobres en nutrientes.

Pero la acumulación de carbono en suelo guarda también relación con el aporte de los excrementos sólidos y el estiércol del ganado, vía por la que retorna una parte del carbono con- 
sumido a pastoreo (SousSANA et al., 2010). Las deyecciones sólidas del ganado y los estiércoles son más resistentes a la descomposición microbiana que los residuos vegetales, ya que están constituidas por la fracción indigestible de la dieta consumida. Experiencias desarrolladas en cultivos agrícolas y forrajeros han revelado que, en una amplia gama de suelos y condiciones climáticas, el almacenamiento de carbono en suelo parece ser mayor cuando se añade estiércol frente a restos vegetales frescos (FRANZLUEBBERS et al., 2008; FrAnZluebBers, 2002). Por tanto, la aplicación de estiércoles es la mejor vía para incorporar materia orgánica y promover el almacenamiento de carbono.

En consecuencia, la clave para mantener o incrementar la acumulación de carbono en los ecosistemas pastorales pasa por mejorar su productividad y por potenciar su aprovechamiento a través del pastoreo directo. Sin embargo, se hace necesario considerar el coste de emisión de carbono que tienen las distintas tecnologías de mejora que, en algunos casos, puede dar lugar a que el sistema se comporte como una fuente en lugar de un sumidero de carbono. En este sentido, una gestión extensiva del ganado puede aportar un mejor balance en el objetivo de capturar carbono aunque no se logren incrementos de producción tan grandes como con otras tecnologías de mejora.

El papel que puede jugar la ganadería extensiva en la mitigacion del cambio climático, dependerá también de la dinámica de otros gases de efecto invernadero vinculados a los sistemas ganaderos, como son el metano y el óxido nitroso (SoussanA et al., 2007). Así, mediante la fermentación ruminal se producen pérdidas del carbono ingerido en forma de metano y con la fertilización nitrogenada (pensemos en el reciclaje en forma de urea) se estimula la producción de óxido nitroso. Hay pocos trabajos que evalúen el papel del ganado en el balance global de estos tres gases, pero parece que la ganadería extensiva puede contribuir a la mitigación del cambio climático principalmente por el secuestro de carbono en los suelos de los terrenos que pastorea. En cualquier caso, es importante que la explotación ganadera sea equilibrada, extensiva para que no se produzcan situaciones de sobrepastoreo que incrementen los riesgos de erosión y por tanto una pérdida del suelo y del carbono en él acumulado.

\section{GANADERÍA EXTENSIVA Y CONSERVACIÓN DE LOS ECOSISTEMAS FORESTALES. LA PREVENCIÓN DE LOS INCENDIOS FORESTALES}

Aunque el fuego constituye una perturbación más que desde siempre ha estado ligada a la dinámica natural de los ecosistemas forestales y ha modelado nuestros paisajes, es cierto que constituye una amenaza para su conservación. De hecho, los incendios forestales son una de las mayores preocupaciones de la sociedad en materia de conservación de los ecosistemas que trasciende más allá de la propia política forestal, dada su repercusión en otras actividades económicas del medio rural y en la protección civil. $\mathrm{La}$ defensa del monte frente a los incendios es uno de los ejes principales de actuación de las administraciones con competencia en medio ambiente, que suelen destinar un presupuesto elevado a tareas de prevención, extinción y restauración.

La utilización del pastoreo en la prevención de los incendios está actualmente en auge y distintos gobiernos autonómicos, entre los que se encuentran la Comunidad Valenciana, Andalucía, Aragón y Cataluña han puesto en marcha programas de distinta naturaleza tendentes a integrar el pastoreo del ganado en los planes de prevención de los incendios forestales de los Montes Públicos. En Andalucía funciona desde el año 2005 la Red de Área Pasto Cortafuego (RAPCA) que ha ido aumentando desde su creación, tanto el número de pastores como las zonas y superficie de actuación. Según la Consejería de Agricultura, Pesca y Medio Ambiente de la Junta de Andalucía, para el año 2013 la RAPCA contará con la participación de 228 pastores que controlarán la vegetación en $3.252 \mathrm{~km}$ lineales de cortafuego en Montes Públicos.

El ganado a pastoreo al consumir hierba, ramón y fruto, disminuye la carga de combustible del monte limpiado el terreno y reduciendo la probabilidad, la frecuencia y la intensidad de los incendios. Cuando el pastoreo se realiza en las áreas cortafuego, el ganado puede mantenerlas con poca vegetación más tiempo, distanciándose las necesidades de intervención mecánica y por tanto rebajándose el coste de su mantenimiento. Por ejemplo, el trabajo de VARELA-REDONDO et 
al. (2008) estima que el coste de eliminación de la vegetación en las áreas cortafuego pueden reducirse sustancialmente si se incorpora el pastoreo del ganado, suponiendo la estrategia combinada (eliminación mecánica más pastoreo) un $36,5 \%$ del coste que implica el empleo de medios mecánicos exclusivamente. Salvo en algunos casos, el pastoreo del ganado en exclusiva no puede mantener operativa un área cortafuego y por tanto su utilización no implica que desaparezca la necesidad de los desbroces mecánicos.

Dado que el objetivo principal es la reducción de la carga de combustible, el pastoreo en las áreas cortafuego suele conducirse de forma intensiva, con una presión de pastoreo elevada, pastando como no se suele hacer cuando lo que se pretende es conservar los pastos. Se busca también en esta zona potenciar el pisoteo del ganado para poder quebrar y reducir físicamente el tamaño de las plantas o las fracciones poco consumidas. Esto puede ser especialmente útil al final de la primavera cuando muchas especies de los pastos se han lignificado, han perdido flexibilidad y son más quebradizas (lo que las hace más susceptibles al pisoteo). Por eso tiene interés trabajar con cabañas numerosas, buscando el efecto rebaño.

Pero para que el pastoreo en el área cortafuego sea posible y pueda realizarse de forma eficiente, los ganaderos deben tener a disposición para su ganado otros pastos en las proximidades, donde el pastoreo ya no se conduce de forma intensiva, sino de acuerdo con la capacidad de pastoreo de los pastos. Por ejemplo, en la red RAPCA estas zonas se denominan respectivamente zonas estratégicas y zonas de refuerzo y generan un gradiente deseado de intensidad de pastoreo (Ruíz-MiRAzo et al., 2009).

Aunque el éxito de muchos de estos programas radica en la existencia de distintos tipos de compensación a los ganaderos por los servicios prestados (JIMÉnEZ et al., 2011), de cara a su mantenimiento en el futuro, sería deseable trabajar en la incorporación de mejoras pastorales en el entorno de las áreas cortafuego que puedan repercutir positivamente en la rentabilidad de estas explotaciones ganaderas y sobre todo en la calidad de vida de los pastores y ganaderos.

Por ejemplo, el aumento de la densidad de abrevaderos en el entorno de los cortafuegos, la fertilización de pastos próximos o el estableci- miento de cultivos forrajeros donde la pendiente y el suelo lo permitan. Además de mejorar la capacidad del ganado para controlar la vegetación, podrían reducir el gasto de compra de piensos y forrajes y mejorar el nivel de alimentación del rebaño y por tanto sus producciones. Por otro lado, la dotación a las áreas cortafuegos de una red de cercados (cercados fijos sobre los que apoyar otros móviles electrificados) aliviaría el trabajo de los pastores que en muchos casos deben permanecer con sus animales largas jornadas diarias. Junto a esto, la diferenciación de estos productos (quizá no por el propio producto, sino por la labor de protección de los ecosistemas forestales que hay detrás de su obtención) y la apertura de nuevas formas de comercialización permitirían avanzar en la rentabilidad de estas explotaciones.

Por último, teniendo en cuenta que las quemas de pastos intencionadas son la principal causa de incendios, la incorporación planificada del pastoreo del ganado en la gestión de los terrenos forestales y en las tareas de control de la vegetación, constituye al mismo tiempo un avance en prevención social (Ruíz-MirAzo et al., 2009). El cuidado diario que necesita el ganado implica una vigilancia continua del territorio, contribuyendo los pastores y ganaderos a la detección con rapidez de cualquier anomalía. En esta misma línea, corresponsabiliza a los pastores y ganaderos locales en la gestión de su propio territorio y por tanto, los hace partícipe de la construcción de su futuro.

\section{A MODO DE CONCLUSIÓN}

La ganadería extensiva modifica algunas propiedades y funciones de los ecosistemas forestales y, bajo determinadas fórmulas de gestión, repercute positivamente en los servicios de regulación que la sociedad demanda de estos ecosistemas.

El papel del ganado en la regulación del ciclo del agua deriva de una compleja interacción de múltiples factores relacionados con su impacto sobre la estructura, composición y pautas de crecimiento de la vegetación, y sobre las propiedades físicas y químicas del suelo. La información disponible parece indicar que el pastoreo puede aumentar la cosecha de agua a nivel de cuenca y puede reducir el drenaje en profundidad a nivel de parcela. En relación al ciclo de los nutrientes, el 
ganado acelera las tasas de conversión desde formas orgánicas hacia formas inorgánicas y este proceso de mineralización puede ser crítico para la producción de muchos ecosistemas forestales y para su capacidad de secuestrar carbono en el suelo. Especialmente relevante en algunos ecosistemas parece ser la contribución del ganado (y no sólo de su pastoreo) a la dinámica del $\mathrm{N}$ a corto plazo, poniendo de manifiesto el papel dual que desempeña como consumidor y descomponedor. El pastoreo afecta a la diversidad funcional de los ecosistemas favoreciendo a las especies anuales y a las que poseen hábito de crecimiento postrado, arquitectura en roseta o estolonífera, y pequeña talla. No obstante, la historia de pastoreo y el contexto climático pueden interferir en este modelo general de respuesta. Si bien a escala de parcela el ganado puede desplazar algunos grupos funcionales, a escala de paisaje los gradientes de utilización pastoral generados por la propia topografía, los tipos de vegetación y la gestión de los ganaderos y pastores, permiten la coexistencia.

Con el abandono de la actividad ganadera y la desaparición de los pastores no sólo disminuye la capacidad de los ecosistemas forestales de ofrecer productos, paisajes y elementos culturales a la sociedad, sino que también se ven afectadas distintas funciones ecosistémicas y, por tanto, desde una perspectiva antropocéntrica la capacidad que tienen de prestar los servicios de regulación que la sociedad demanda actualmente. Por tanto, la integridad de muchos de nuestros ecosistemas forestales y su capacidad de generar servicios de regulación precisan de la incorporación planificada del pastoreo del ganado.

\section{BIBLIOGRAFÍA}

Aires, L.M.; Pio C.A. \& PereirA, J.S.; 2008. The effect of drought on energy and water vapour exchange above a mediterranean $\mathrm{C} 3 / \mathrm{C} 4$ grassland in Southern Portugal. Agric. Forest Meteorol. 148: 565-579.

AldeZabal, A.; García-GonZÁlez, R.; GómeZGarcía, D. y Fillat, F.; 2002. El papel de los herbivoros en la conservacion de los pastos. Ecosistemas 2002(3).

ANDERSON, V.J. \& BRISKE, D.D.; 1995. Herbivore-induced species replacement in grasslands: is it driven by herbivory tolerance or avoidance? Ecol. Appl. 5: 1014-1024.

ARCHER, S.; 1995. Herbivore mediation of grass-woody plant interactions. Trop. Grassl. 29: 218-235.

Augustine, D.J. \& Mcnaughton, S.J.; 2006. Interactive effects of ungulate herbivores, soil fertility and variable rainfall on ecosystem processes in a semi-arid savanna. Ecosystems 9: 1242-1256.

Augustine, D.J.; 2003a. Long-term, livestockmediated redistribution of nitrogen and phosphorous in an East African savanna. $J$. Appl. Ecol. 40: 137-49.

Augustine, D.J.; 2003b. Spatial heterogeneity in the herbaceous layer of a semi-arid savanna ecosystem. Plant. Ecol. 167: 319-332.

Bell, L.W.; KIRKEgaARD, J.A.; SwAN, A.; HunT, J.R.; Huth, N.I. \& FetTELl, N.A.; 2011. Impacts of soil damage by grazing livestock on crop productivity. Soil. Till. Res. 113: 19-29.

Bengough, A.G.; Mckenzie, B.M.; Hallett, P.D. \& VAlEnTine, T.A.; 2011. Root elongation, water stress, and mechanical impedance: a review of limiting stresses and beneficial root tip traits. J. Exp. Bot. 62: 59-68.

Bilotta, G.S.; BrazIER, R.E. \& HAYGaRTH, P.M.; 2007. The impacts of grazing animals on the quality of soils, vegetation, and surface waters in intensively managed grasslands. Adv. Agron. 94: 237-280.

Blunden, B.G.; Mcbride, R.A.; Daniel, H. \& BLACKWELL P.S.; 1994. Compactation of an earthy sand by rubber tracked and tyred vehicles. Australian J. Soil Res. 32: 1095-1108.

BRISKE, D.; 1996 Strategies of plant survival in grazed systems: a functional interpretation. In: J. Hodgson \& A. Illius (eds.), The Ecology and Management of Grazed Systems: 37-67. $\mathrm{CAB}$ International. Wallingford.

CARBONERO, M.D.; FAJARDO, E.; GUERRERO, J.E.; García, A. y FernándeZ-Rebollo, P.; 2013. Pastoreo en olivares: producción ganadera y efectos sobre los costes de cultivo. En: L. Olea, M.J. Poblaciones, S. Rodrigo y O. Santamaría (eds), Actas de la 52 Reunión Científica de la SEEP. Los pastos: nuevos retos, nuevas oportunidades: 513-520. Badajoz.

Castro, J. y Robles, AB.; 2003. Dispersión endozoócora por ganado ovino de las semi- 
llas de seis especies cistáceas. En: A.B. Robles, M.E. Ramos, C. Morales, E. de Simón, J.L. \& González-Rebollar, J. Boza (eds), Actas de la XLIIV Reunión científica de la SEEP. Pastos, desarrollo y conservación: 645-650. Granada.

Cernusca, A.; Bahn, M.; Berninger, F.; TAPPeINER, U. \& WOHLFAHRT, G.; 2008. Effects of Land-Use Changes on Sources, Sinks and Fluxes of Carbon in European Mountain Grasslands. Ecosystems 11: 1335-1337.

Daniel, J.A.; Potter, K.; Altom, W.; Aljoe, H. \& Stevens, R.; 2002. Long-term grazing density impacts on soil compaction. Transactions of the ASAE. 45: 1911-1915.

De Bello, F.; LePs, J. \& Sebastia, M.T.; 2006. Variations in species and functional plant diversity along climatic and grazing gradients. Ecography 29: 801-810.

Díaz, S.; Lavorel, S.; Mcintyre, S.; FalczuK, V.; Casanoves, F.; Milchunas, D.G.; Skarpe, C.; Rusch, G.; Sternberg, M.; Noy-MeIr, I.; LANDSBerg, J.; Zhang, W.; Clark, H. \& CAmpbell, B.D.; 2007. Plant trait responses to grazing -a global synthesis. Global Change Biology 13: 313-341.

Donkor, N.T.; Gedir, J.V.; Hudson, R.J.; Bork, E.W.; ChanasyK, D.S. \& NAETH, M.A.; 2002. Impacts of grazing systems on soil compaction and pasture production in Alberta. Can. J. Soil Sci. 82: 1-8.

DREWRY, J.J.; 2006. Natural recovery of soil physical properties from treading damage of pastoral soils in New Zealand and Australia: A review. Agr. Ecosyst. Environ. 114: 159-169.

Drewry, J.J.; Cameron, K.C. \& Buchan, G.D.; 2008. Pasture yield and soil physical property responses to soil compaction from treading and grazing - a review. Aust. J. Soil. Res. 46: 237-256.

Drewry, J.J.; Paton, R.J. \& Monaghan, R.M.; 2004. Soil compaction and recovery cycle on a Southland dairy farm: implications for soil monitoring. Aust. J. Soil Res. 42: 851-856.

DYKSTERHUIS, E.J.; 1949. Condition and management of rangelands based on quantitative ecology. J. Range Manage. 2: 104-115.

FANLO, R. y ChOCARRo, C.; 2013. Parámetros topográficos y de gestión que influyen en la producción y calidad forrajera en prados de montaña catalanes. En: L. Olea, M.J. Poblaciones, S. Rodrigo y O. Santamaría (eds.), Actas de la 52 Reunión Científica de la SEEP. Los pastos: nuevos retos, nuevas oportunidades: 481-488. Badajoz.

FERnÁndeZ-REBollo, P.; BláZqueZ, A.; AGÜERA, J.; LECHUGA, M.P. y CARBONERO, M.D.; 2004. Efecto del pastoreo con ganado ovino y el laboreo en las propiedades fisicas y químicas de un suelo de textura arenosa de dehesa. En: B. García, A. García, B. Vázquez de Aldana, I. Zabalgogeazcoa (eds.), Actas de la XLIV Reunión Científica de la SEEP. Pastos y ganadería extensiva: 721-727. Salamanca.

FranZluebBers, A.J. \& Arshad, M.A.; 1996. Soil organic matter pools during early adoption of conservation tillage in northwestern Canada. Soil Sci. Soc. Am. J. 60: 1422-1427.

Franzluebbers, A.J. \& Stuedemann, J.A.; 2008. Soil physical responses to cattle grazing cover crops under conventional and no tillage in the Southern Piedmont USA. Soil. Till. Res. 100: 141-153.

FRANZLUEBBERS, A.J.; 2002. Soil organic matter stratification ratio as an indicator of soil quality. Soil. Till. Res. 66: 95-106.

García-Moreno, A.; Carbonero, M.D., SerraNo Moral, M. y Fernández-Rebollo, P.; 2014. Grazing effects shoot growth and water status of Quercus ilex L. in Mediterranean open woodlands. Ann. For. Sci. 71: 917-926.

García-Moreno, A.; CARbonero, M.D.; Moreno, F.; Leal, J.R.; Hidalgo, M.T. \& FERnÁndeZ-Rebollo, P.; 2013. Efecto del pastoreo en la producción de bellotas de la encina en la dehesa. Consecuencias para el follaje del árbol. En: L. Olea, MJ. Poblaciones, S. Rodrigo, O. Santamaría (eds.), Actas de la 52 Reunión Científica de la SEEP. Los pastos: nuevos retos, nuevas oportunidades: 537-544. Badajoz.

GreENwood, K.L. \& MCKENZIE, B.M.; 2001. Grazing effects on soil physical properties and the consequences for pastures: a review. Austral. J. Exp. Agric. 41: 1231-1250.

Grime, J.P.; 2001. Plant Strategies, Vegetation Processes, and Ecosystem Properties. 2nd edition. John Wiley and Sons. Chichester.

HerReRA, C.M.; 2000. Dispersión de semillas por animales en el Mediterráneo: ecología y evo- 
lución. En: R. Zamora \& F.I. Puignaire (eds), Ecosistemas Mediterráneos. Análisis funcional. CSIC-AEET, Textos Universitarios $\mathrm{n}^{\circ} 32$.

Houlbrooke, D.J.; Paton, R.J.; LitTlejohn, R.P. \& MORTON, J.D.; 2011. Land-use intensification in New Zealand: effects on soil properties and pasture production. J. Agr. Sci. 149: 337-349.

Jiménez, R.; Mena, Y.; CASTel, J.M. y Ruiz, F.; 2011. Caracterización técnica y productiva de las explotaciones de pequeños rumiantes implicadas en la red de áreas pasto-cortafuegos de Andalucía (RAPCA). Cuad. Soc. Esp. Cienc. For. 33: 131-136.

Kulli, B.; Gysi, M. \& Fluhler, H.; 2003. Visualizing soil compaction based on flow pattern analysis. Soil. Till. Res. 70: 29-40.

LeChuga, M.P.; 2005. Efectos del laboreo y el pastoreo en las características del suelo y el pasto de las dehesas. Implicaciones para la regeneración del arbolado. Trabajo Profesional Fin de Carrera. ETSI Agrónomos y Montes, Universidad de Córdoba.

LEE, T.D.; ReICH, P.B. \& TJoELKer, M.G.; 2003. Legume presence increases photosynthesis and $\mathrm{N}$ concentrations of co-occurring nonfixers but does not modulate their responsiveness to carbon dioxide enrichment. Ecophysiology 137: 22-31.

MARAÑON, T.; 1986. Plant species richness and canopy effect in the savanna-like "dehesa" of S.W. Spain. Ecologia Mediterranea 12: 131-141.

Materechera, S.A.; DeXter, A.R. \& Alston, A.M.; 1991. Penetration of very strong soils by seedling roots of different plant-species. Plant Soil. 135: 31-41.

Milchunas, D.G.; Sala, O.E. \& Lauenroth, W.K.; 1988. A generalized model of the effects of grazing by large herbivores on grassland community structure. The American Naturalist 132: 87-106.

OlfF, H. \& RitchiE, ME.; 1998. Effects of herbivores in grassland plant diversity. Trends Ecol. Evol. 13: 261-265.

Parras-Alcántara, L.; DíaZ-Jaimes, L.; Lozano-García, B.; Fernández-Rebollo, P.; Moreno-Elcure, F. \& CARboneroMuÑOZ, M.D.; 2014. Organic Farming has little effect on carbon stock in a
Mediterranean dehesa (Southern Spain). Catena 113: 9-17.

Peco, B.; Sánchez, A.M. y Azcarate, F.M.; 2006. Abandonment in grazing systems: Consequences for vegetation and soil. Agr. Ecosyst. Environ. 113: 284-294.

Ritchie, M.E.; Tilman, D. \& KNOPS, J.M.H.; 1998. Herbivore effects on plant and nitrogen dynamics in oak savanna. Ecology 79: 165-77.

RoBles, AB.; 2008. En el conjunto de las Sierras Béticas: Pastos, producción, diversidad y cambio global. En: P. Fernandez, A. Gómez, J.E. Guerrero, A. Varo, C. Calzado, A. García, M.D. Carbonero, A. Blazquez, S. Escuin y S. Castillo (eds.), Actas de la XLVIII Reunión Científica de la SEEP. Pastos, clave en la gestión de los territorios: Integrando disciplinas: 31-52. Córdoba.

RodríGUEZ-MurILlo, J.C.; 2001. Organic carbon content under different types of land use and soil in peninsular Spain. Biology and Fertility of Soils 33: 53-61.

Ruiz-Mirazo, J.; Robles, A.B. \& GonzÁlezRebollar, J.L.; 2009. Pastoralism in Natural Park of Anadalusía (Spain): a tool for fire prevention and the naturalization of ecosystems. Options Méditerranéennes 91: 141-144.

Schnabel, S.; Pulido-Fernández, M. \& LAVADO-CONTADOR, J.F.; 2013. Soil water repellency in rangelands of Extremadura (Spain) and its relationship with land management. Catena 103: 53-61.

Sebastia, M.T.; De Bello, F.; Puig, L. \& TAULL, M.; 2008. Grazing as a factor structuring grasslands in the Pyrenees. Appl. Veg. Sci. 11: 215-223.

Sebastia, M.T.; Palero, N. \& De Bello, F.; 2011. Changes in management modify agrodiversity in sainfoin swards in the Eastern Pyrenees. Agron. Sustain. Dev. 31: 533-540.

Seddaiu, G.; Porcu, G.; LeddA, L.; Roggero, P.P.; Agnelli, A. \& Corti, G.; 2013. Soil organic matter content and composition as influenced by soil management in a semiarid Mediterranean agro-silvo-pastoral system. Agr. Ecosyst. Environ. 167: 1-11.

SoANE, B.D.; 1990. The role of organic-matter in soil compactibility - a review of some practical aspects. Soil. Till. Res. 16: 179-201. 
SoussanA, J.F.; (27 autores más); 2007. Full accounting of the greenhouse gas $(\mathrm{CO} 2, \mathrm{~N} 2 \mathrm{O}$, $\mathrm{CH} 4$ ) budget of nine European grassland sites. Agr. Ecosyst. Environ. 121: 121-134.

Soussana, J.F.; TAlleC, T. \& Blanfort, V.; 2010. Mitigating the greenhouse gas balance of ruminant production systems through carbon sequestration in grasslands. Animal 4: 334-350.

StaVi, I.; LaL, R. \& Owens, L.B.; 2011. Effects of cattle grazing during the dormant season on soil surface hydrology and physical quality in a moist-temperate region. Ecohydrology 4: 106-114.

STOCK, O. \& Downes, N.K.; 2008. Effects of additions of organic matter on the penetration resistance of glacial till for the entire water tension range. Soil. Till. Res. 99: 191-201.
Tarrega, R.; Calvo, L.; TABoada, A., GarcíaTejero, S. \& Marcos, E.; 2009. Abandonment and management in Spanish dehesa systems: Effects on soil features and plant species richness and composition. Forest Ecol. Manage. 257: 731-738.

Terradillos, A.; 1997. Parque Natural de Cabo de Gata-Nijar. Análisis del sistema ganadero. Trabajo Profesional Fin de Carrera. ETSI Agrónomos y Montes, Universidad de Córdoba.

Varela, E.; Calatrava, J.; Ruiz-Mirazo, J.; JiMÉNEZ，R. Y GONZÁLEZ-REBOLLAR，J.L.; 2008. El pastoreo en la prevención de incendios forestales: análisis comparado de costes evitados frente a medios mecánicos de desbroce de la vegetación. Pequeños Rumiantes 9(3): 12-20. 